Louisiana State University

LSU Digital Commons

Faculty Publications

Department of Physics \& Astronomy

7-13-1998

\title{
Temporal coherence of ultrashort high-order harmonic pulses
}

M. Bellini

LENS - European Laboratory for Non-Linear Spectroscopy

C. Lyngå

Lunds Tekniska Högskola

A. Tozzi

Università degli Studi di Firenze

M. B. Gaarde

Lunds Tekniska Högskola

T. W. Hänsch

Istituto Nazionale per la Fisica della Materia

See next page for additional authors

Follow this and additional works at: https://digitalcommons.Isu.edu/physics_astronomy_pubs

\section{Recommended Citation}

Bellini, M., Lyngå, C., Tozzi, A., Gaarde, M., Hänsch, T., L'Huillier, A., \& Wahlström, C. (1998). Temporal coherence of ultrashort high-order harmonic pulses. Physical Review Letters, 81 (2), 297-300.

https://doi.org/10.1103/PhysRevLett.81.297

This Article is brought to you for free and open access by the Department of Physics \& Astronomy at LSU Digital Commons. It has been accepted for inclusion in Faculty Publications by an authorized administrator of LSU Digital Commons. For more information, please contact ir@lsu.edu. 
Authors

M. Bellini, C. Lyngå, A. Tozzi, M. B. Gaarde, T. W. Hänsch, A. L'Huillier, and C. G. Wahlström

This article is available at LSU Digital Commons: https://digitalcommons.Isu.edu/physics_astronomy_pubs/2189 


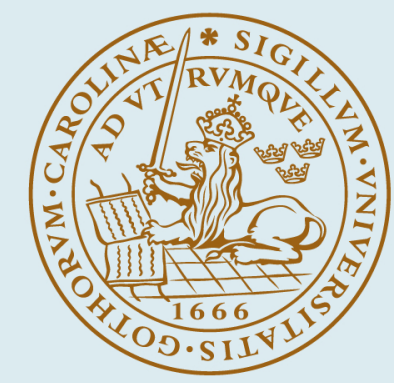

\title{
LUND UNIVERSITY
}

\section{Temporal coherence of ultrashort high-order harmonic pulses}

\author{
Bellini, M; Lynga, C; Tozzi, A; Gaarde, M. B; Hansch, T. W; L'Huillier, Anne; Wahlström, \\ Claes-Göran \\ Published in: \\ Physical Review Letters
}

DOI:

10.1103/PhysRevLett.81.297

1998

Link to publication

Citation for published version (APA):

Bellini, M., Lynga, C., Tozzi, A., Gaarde, M. B., Hansch, T. W., L'Huillier, A., \& Wahlström, C-G. (1998).

Temporal coherence of ultrashort high-order harmonic pulses. Physical Review Letters, 81(2), $297-300$. https://doi.org/10.1103/PhysRevLett.81.297

\section{Total number of authors:}

7

\section{General rights}

Unless other specific re-use rights are stated the following general rights apply:

Copyright and moral rights for the publications made accessible in the public portal are retained by the authors and/or other copyright owners and it is a condition of accessing publications that users recognise and abide by the legal requirements associated with these rights.

- Users may download and print one copy of any publication from the public portal for the purpose of private study or research.

- You may not further distribute the material or use it for any profit-making activity or commercial gain

- You may freely distribute the URL identifying the publication in the public portal

Read more about Creative commons licenses: https://creativecommons.org/licenses/

Take down policy

If you believe that this document breaches copyright please contact us providing details, and we will remove

access to the work immediately and investigate your claim.
} 


\title{
Temporal Coherence of Ultrashort High-Order Harmonic Pulses
}

\author{
M. Bellini, ${ }^{1,2}$ C. Lyngå, ${ }^{3}$ A. Tozzi, ${ }^{4}$ M. B. Gaarde, ${ }^{3,5}$ T. W. Hänsch, ${ }^{2,4,6}$ A. L’Huillier, ${ }^{3}$ and C.-G. Wahlström ${ }^{3}$ \\ ${ }^{1}$ European Laboratory for Non Linear Spectroscopy (L.E.N.S.), Largo E. Fermi, 2, I-50125 Florence, Italy \\ ${ }^{2}$ Istituto Nazionale di Fisica della Materia, INFM, Florence, Italy \\ ${ }^{3}$ Department of Physics, Lund Institute of Technology, P.O. Box 118, S-221 00 Lund, Sweden \\ ${ }^{4}$ Department of Physics, University of Florence, Largo E. Fermi, 2, I-50125 Florence, Italy \\ ${ }^{5}$ Niels Bohr Institute, Ørsted Laboratory, 2100 Copenhagen, Denmark \\ ${ }^{6}$ Max-Planck-Institut für Quantenoptik, P.O. Box 1513, D-85740 Garching, Germany
}

(Received 19 December 1997)

\begin{abstract}
We have studied the temporal coherence of high-order harmonics (up to the 15th order) produced by focusing $100 \mathrm{fs}$ laser pulses into an argon gas jet. We measure the visibility of the interference fringes, produced when two spatially separated harmonic sources interfere in the far field, as a function of the time delay between the two sources. In general, we find long coherence times, comparable to the expected pulse durations of the harmonics. For some of the harmonics, the interference pattern exhibits two regions, with significantly different coherence times. These results are interpreted in terms of different electronic trajectories contributing to harmonic generation. [S0031-9007(98)06569-7]
\end{abstract}

PACS numbers: $32.80 . \mathrm{Rm}, 42.65 . \mathrm{Ky}$

The generation of high-order harmonics of a short laser pulse in a gas jet has attracted a lot of attention since the first observations. Besides its fundamental interest as a signature of the interaction between an atom and a strong radiation field, the harmonic radiation has the potential to become a useful short-pulse coherent source of light in the extreme ultraviolet (XUV) region. During the past few years, the generation process has been thoroughly studied as a function of various parameters [1]. The radiation has been characterized in certain conditions, both spatially [2-5], and temporally [6,7]. However, the temporal coherence which is a very fundamental property, has gone largely unexplored so far, except for some spectral measurements [8]. Recently, we have shown that two spatially separated sources of high harmonic radiation created by the same laser pulse are phase locked [9] and lead to interference fringes when superposed in the far field. That experiment, however, did not shed light on the temporal coherence of the harmonic radiation, since the two harmonic pulses were superposed in time.

There are several potential causes of degradation of the temporal coherence of the high-order harmonics. One is the ionization of the medium, leading to a "chirp" (or frequency modulation) of the fundamental laser pulse and consequently of the harmonics. Another mechanism is the rapid intensity-dependent variation of the phase of the high-order harmonics predicted in the single atom response: Since the atom experiences different intensities as a function of time during the laser pulse, this may lead to a frequency chirp of the generated field $[10,11]$. The experimental investigation of the temporal coherence of high-order harmonics is of crucial importance, both from a fundamental point of view, to confirm or not different theoretical models, as well as for applications such as spectroscopy, interferometry, and holography in the XUV region.
In this Letter, we report the first direct measurements of the temporal coherence of high-order harmonics of a short-pulse laser. The experimental setup, an extension of that used for a preliminary experiment with the third harmonic [12], is shown in Fig. 1.

Briefly, the laser is an amplified titanium-sapphire system delivering $100 \mathrm{fs}$ pulses in a $14 \mathrm{~nm}$ bandwidth centered around $790 \mathrm{~nm}$ at a $1 \mathrm{kHz}$ repetition rate and with an energy up to $0.7 \mathrm{~mJ}$. A Michelson interferometer, placed in the path of the laser beam, produces pairs of infrared pump pulses having equal intensities and whose relative delay can be accurately adjusted by means of a computercontrolled stepping motor. The beams are then apertured down to a diameter of $6 \mathrm{~mm}$ by an iris and focused by a $200 \mathrm{~mm}$ focal length lens into the pulsed argon gas jet produced by a fast piezoelectric valve. After the iris and for each infrared pulse, we measure an energy of $0.1 \mathrm{~mJ}$ and a pulse duration of $100 \mathrm{fs}$, giving a peak intensity in the focus of the order of $10^{14} \mathrm{~W} / \mathrm{cm}^{2}$. In order to avoid interference effects in the focal zone and to prevent perturbations of the medium induced by the first pulse, we have slightly misaligned one arm of the interferometer, so that the paths of the two pulses are not perfectly parallel to each other and form a focus in two separate positions of the jet (separated by about $50 \mu \mathrm{m}$ ). Both pulses then interact with "fresh" atoms and produce harmonics as two separate and independent sources that may interfere in the far field. The jet is placed at the position of the entrance slit of a $1 \mathrm{~m}$ vacuum UV monochromator, equipped with a normal incidence spherical grating with 600 lines $/ \mathrm{mm}$. About $50 \mathrm{~cm}$ after the exit slit of the monochromator, the spectrally separated but spatially overlapped beams are detected on the sensitive surface of a microchannelplate detector (MCP) coupled to a phosphor screen and a CCD camera. 


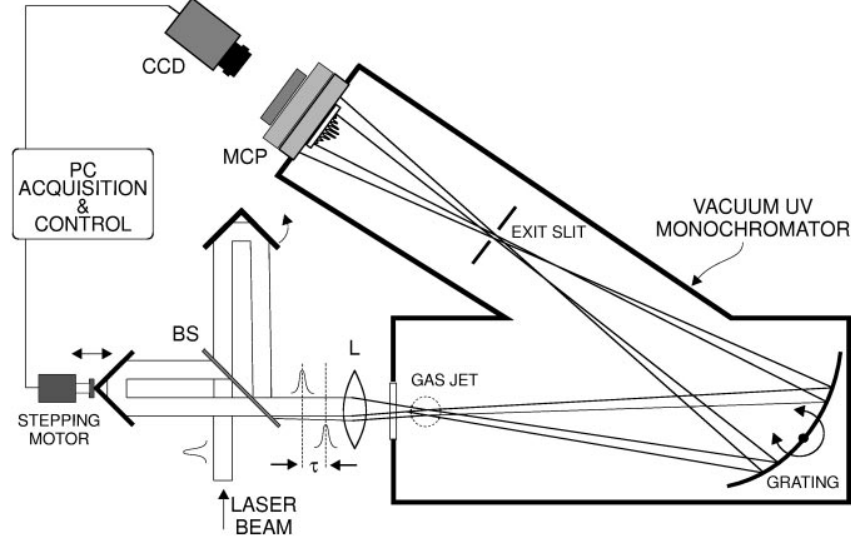

FIG. 1. Experimental setup. BS is a broadband $50 \%$ beam splitter for $800 \mathrm{~nm}$. L is the lens used to focus the two pulses, separated by the time delay $\tau$, into the gas jet.

It is interesting at this point to emphasize the differences and similarities of our setup with a Michelson interferometer (used in measurements of temporal coherence) or with Young's double-slit experiment (used in measurements of spatial coherence). In Young's experiment, the two secondary sources created at the positions of the slits originate as two different portions of the same wave front. The visibility of the fringes observed is then a measure of the spatial coherence of the primary source. In our setup, there are two independent sources of harmonic radiation placed at the locations of the "slits." Therefore, we are not probing the spatial coherence, but rather the phase locking between the two sources as a function of the temporal delay. The distance between the two sources is very small compared to the distance of observation, so the spatial distributions in the far field originating from the two sources are practically overlapped. This means that we are probing the correlation (in time) between the same spatial areas in the two beams. In Michelson's experiment, the splitting into two pulses with a variable time delay is done on the analyzed radiation. A detector is recording the light intensity modulations (at a given spatial point), as a function of the time delay between the two pulses. In our setup, the splitting into two pulses with a variable time delay is done on the infrared pump radiation. The two pulses are then focused at slightly different positions to produce two harmonic pulses. This is an extremely simple way to produce two time-delayed pulses in the XUV region, where it would be difficult to find good optics for the beam splitter and mirrors. The visibility of the fringes is obtained spatially by analyzing the far-field patterns, which is equivalent to selecting a given space point and fine-tuning the time delay, since two different space points in the far-field pattern correspond to two different time delays between the two pulses.

Let us emphasize that the dispersive diffraction grating used to separate the harmonics does not change the coherence time observed in our experiment. For a given harmonic order, any spectral filtering effects remain negligible as long as the frequency-dependent lateral displacement of the wave fronts is much smaller than the spot size for the fringe pattern. As a test to confirm this, we have measured the coherence time of the third harmonic in two conditions: after zero-order reflection on the grating, and after diffraction in the first order. The results are identical within the experimental error bars.

When the two laser pulses are superposed in time, the far-field pattern shows interference fringes with a relatively high contrast (up to 0.6). This is true for all of the observed harmonics, i.e., from the 7th up to the 21 st. Higher-order harmonics have not been detected due to the low pump intensity available. An example of the recorded far-field interference pattern for the 21st harmonic is shown in Fig. 2. All the images shown are single-shot acquisitions. The fringe patterns show a high stability, only slightly disturbed by residual mechanical vibrations in the interferometer, as well as a high robustness against differences in intensities in the two pump pulses. These observations confirm the results presented in our previous paper [9], and extend them to laser pulses of significantly higher intensity and shorter duration, as well as to shorter generated wavelengths.

To determine the temporal coherence of the high-order harmonics, we vary the time delay in steps of 5 or $10 \mathrm{fs}$ and take successive recordings of the interference patterns. The fringe visibility $V=\left(I_{\max }-I_{\min }\right) /\left(I_{\max }+I_{\min }\right)$ is measured at a given spatial point (in general at the center) for the different delays $\tau$. The coherence time is obtained as the half width at half maximum of the curve $V(\tau)$. The coherence times measured at the center of the spatial profile vary from 20 to $40 \mathrm{fs}$, relatively independently of the harmonic order. Measuring the coherence time is equivalent to determining the spectral width of the radiation, since it is inversely proportional to the bandwidth. For a Fourier-transform-limited Gaussian pulse, the coherence time is equal to the pulse duration. The coherence times mentioned above correspond to bandwidths between 0.1 and $0.2 \mathrm{~nm}$. Determinations of the spectral widths of the

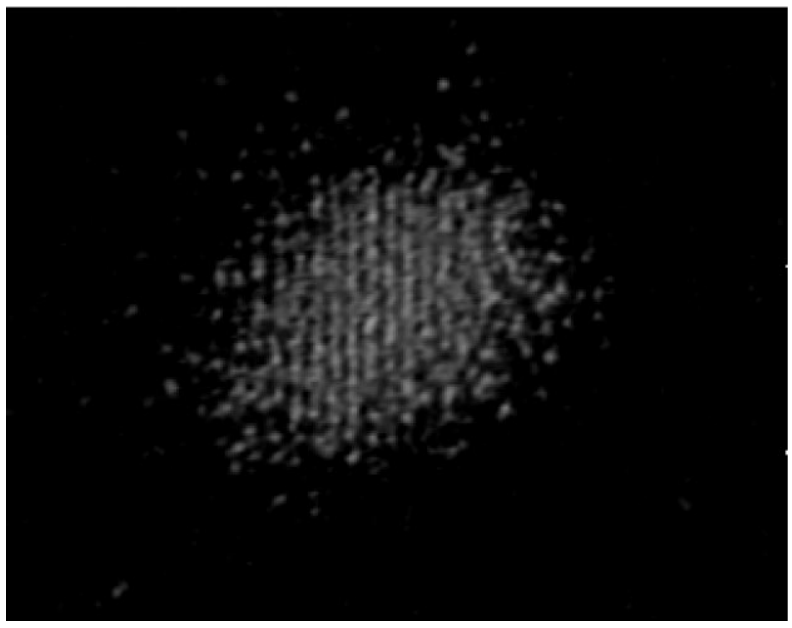

FIG. 2. Interference fringe pattern for the 21 st harmonic. 
harmonics using conventional (diffractive) methods are scarce, probably because the grating spectrometers used in most of the experiments do not have the necessary resolution [8]. In addition, in order to increase the transmission, the diffractive spectrometers used to record the harmonic spectra are often used without entrance slit, so that the spectral width obtained is integrated over the spatial profile. The strength of our method is that we can analyze the spectral content of the harmonics spatially.

In Fig. 3, we show the interference pattern obtained for the 15th harmonic, at two time delays, 0 (a) and $15 \mathrm{fs}$ (b). It consists of two well separated spatial regions, depending on the radial coordinate $r$ : an intense inner region $(r<2 \mathrm{~mm})$ with a long coherence time, and an outer region ( $2 \mathrm{~mm}<r<8 \mathrm{~mm}$ ), containing about $80 \%$ of the total energy, with a much shorter coherence time,
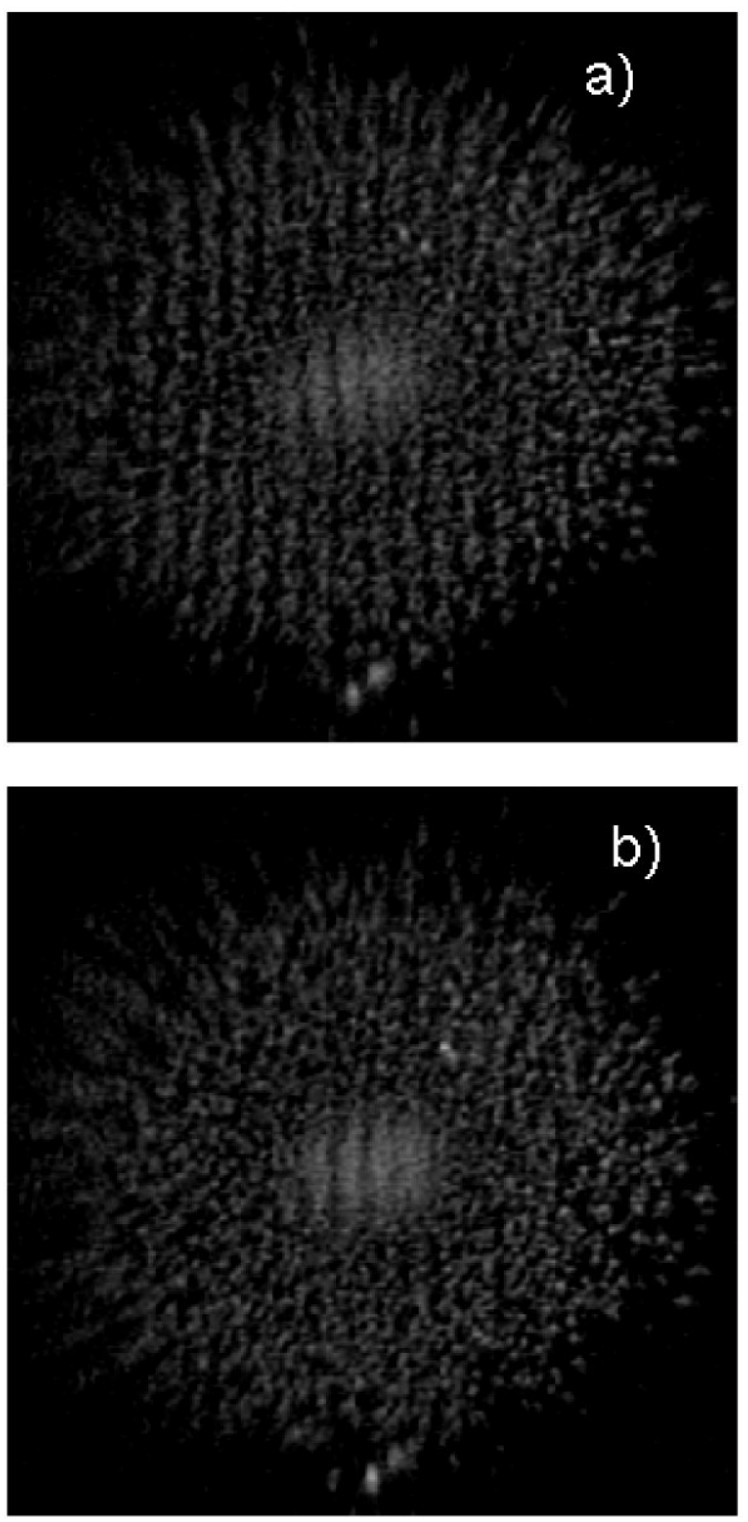

FIG. 3. Interference fringe pattern for the 15th harmonic. (a) $\tau \approx 0$ fs. (b) $\tau \approx 15$ fs. of the order of a few femtoseconds. Although fringes are clearly seen over the entire angular distribution in Fig. 3(a), they disappear in the outer region in Fig. 3(b). The fringe visibilities, measured around $r=0 \mathrm{~mm}$ for the inner region and around $r=5 \mathrm{~mm}$ for the outer region, are reported in Fig. 4(a) as a function of the time delay in full and open circles, respectively.

To understand these results, we have performed numerical simulations, including both single-atom response [11] and propagation, as described in [13]. In Fig. 5, we show calculated fringe patterns for the 15th harmonic, for several time delays, at an intensity of $1.4 \times 10^{14} \mathrm{~W} / \mathrm{cm}^{2}$. As in the experiment, the calculated fringe patterns show two distinct spatial areas: a temporally coherent inner region and an almost incoherent outer region. The visibility obtained in the two regions is plotted in Fig. 4(b) with full and open squares for the inner and outer regions, respectively.

In the semiclassical interpretation of harmonic generation [14], an electron initially in the ground state of an atom, and exposed to an intense, low-frequency, linearly polarized, electromagnetic field, first tunnels through the barrier formed by the Coulomb and the laser fields. When the laser field changes sign, the electron may be driven back towards the core with high kinetic energy and recombine to the ground state, giving rise to emission of high-energy harmonic photons. There are actually
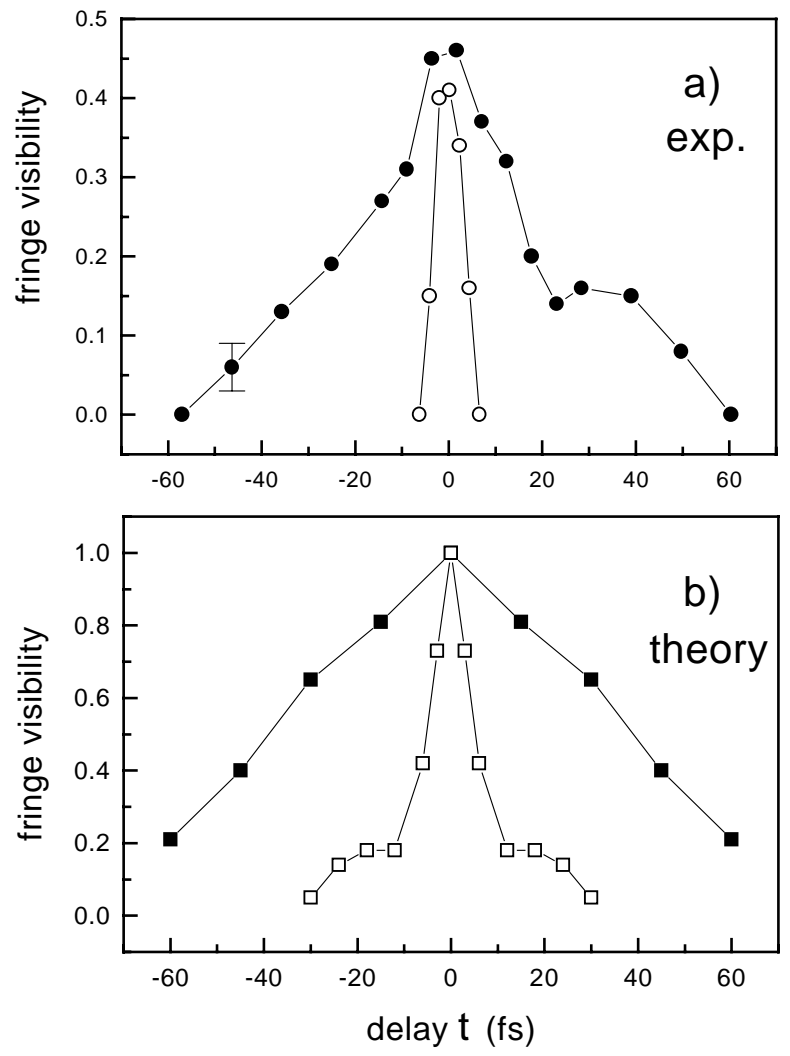

FIG. 4. Visibility curves as a function of the delay for the 15 th harmonic, for the inner (full symbols) and outer (open symbols) regions; (a) experiment; (b) theory. 


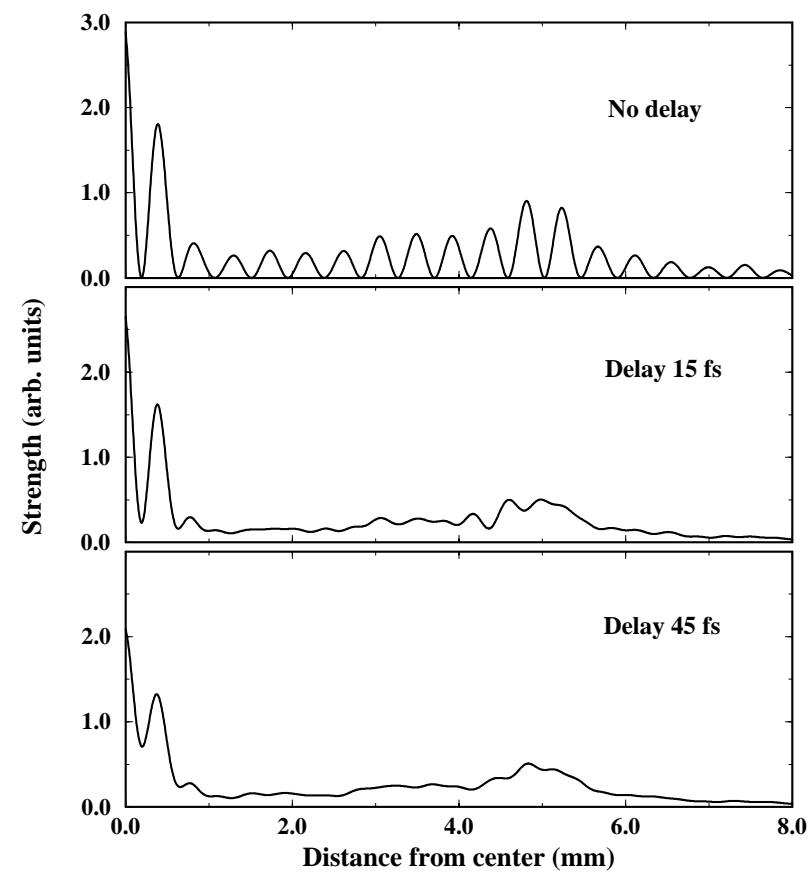

FIG. 5. Calculated fringe patterns for the 15th harmonic, for different time delays.

two main trajectories contributing, with different phase properties, to the dipole moment of some particular harmonic [15]. The contribution of the first, "short," trajectory (excursion time close to half an optical period) has a phase that does not vary much with the laser intensity, whereas the phase corresponding to the second, "long," trajectory (excursion time slightly less than an optical period) varies rapidly with the laser intensity.

In our simulation, we can examine separately the contributions of these trajectories, leading to quite different temporal and spatial characteristics of the emitted radiation. The long electronic trajectory gives rise to strongly divergent angular emission $[8,10]$ : This is because the rapid variation of the dipole phase with the laser intensity $(I)$ leads to a strong curvature of the phase front [which depends on $I(r)$ ]. This radiation has a very short coherence time, since the harmonic pulse is strongly chirped due to the rapid variation of the phase during the pulse [which depends on $I(t)$ ]. In contrast, the phase variation corresponding to the short trajectory is much less important. The emitted radiation has a long coherence time and is much more collimated. By measuring the spectral content (temporal coherence) of the far-field profile of the emitted radiation, we gain insight on the inner dynamics of the atom in the presence of a strong laser field. Finally, note that by simply aperturing the beam, it should be possible to select a highly spatially and temporally coherent radiation, of interest for applications.

In conclusion, we have studied the temporal coherence of high-order harmonics emitted when an intense laser pulse is focused into a jet of argon atoms. The measured coherence times at the center of the angular profile are long, of the order of the expected pulse durations of the harmonics. For some harmonics, two spatial regions of temporal coherence can be observed: an inner region with a long coherence time and an outer one, with extremely short temporal coherence. We interpret the observed spatiotemporal behaviors as the signature of the two main electronic trajectories involved in the harmonic generation process.

The experimental part of this work was carried out at the European Laboratory for Non Linear Spectroscopy (L.E.N.S.), Florence, Italy, with the support of MURST and of European Community Contract No. ERBFMGECT950017. We thank the group of S. Solimeno for lending us the pulsed valve used for the experiment. We also thank K. C. Kulander and K. J. Schafer for the use of their single atom data, and F. Salin and Ph. Balcou for interesting discussions. The support of the Swedish Natural Science Research Council is acknowledged.

[1] See contributions in Proceedings of the 7th International Conference on Multiphoton Processes, Garmisch-Partenkirchen, Germany, 1996, edited by P. Lambropoulos and $\mathrm{H}$. Walther, Institute of Physics Conference Series No. 154 (IOP Publishing, Bristol, Philadelphia, 1996).

[2] P. Salières et al., J. Phys. B 29, 4771 (1996).

[3] J. Peatross and D. D. Meyerhofer, Phys. Rev. A 51, R906 (1995).

[4] J. W. G. Tisch et al., Phys. Rev. A 49, R28 (1994).

[5] T. Ditmire et al., Phys. Rev. Lett. 77, 4756 (1996).

[6] T. E. Glover et al., Phys. Rev. Lett. 76, 2468 (1996).

[7] A. Bouhal et al., J. Opt. Soc. Am. B 14, 950 (1997); (to be published

[8] A review of measurements and calculations of spatiotemporal characteristics of harmonics is presented in P. Salières et al., Adv. At. Mol. Opt. Phys. (to be published).

[9] R. Zerne et al., Phys. Rev. Lett. 79, 1006 (1997).

[10] P. Salières et al., Phys. Rev. Lett. 74, 3776 (1995).

[11] K. J. Schafer and K. C. Kulander, Phys. Rev. Lett. 78, 638 (1997).

[12] M. Bellini and T.W. Hänsch, Appl. Phys. B 65, 677 (1997).

[13] M. B. Gaarde et al., Phys. Rev. A 57, 4553 (1998).

[14] P. B. Corkum, Phys. Rev. Lett. 71, 1994 (1993); K. J. Schafer et al., Phys. Rev. Lett. 70, 1599 (1993).

[15] M. Lewenstein et al., Phys. Rev. A 52, 4747 (1995). 